\title{
Design and Characterisation of a Non-contact Flexible Sensor Array for Electric Potential Imaging Applications
}

\author{
Arash Pouryazdan, Member, IEEE, Julio C. Costa, Student Member, IEEE, \\ Leonardo Garcia-Garcia, Member, IEEE, Pasindu Lugoda, Member, IEEE, Robert J. Prance, \\ Helen Prance, and Niko Münzenrieder, Senior Member, IEEE
}

This report is an extension of the work presented at the IEEE FLEPS 2020 conference titled flexible array of sensing probes for non-contact SEPM applications [1].

A. Pouryazdan, J. C. Costa, L. Garcia-Garcia, P. Lugoda, R. J. Prance, H. Prance and N. Münzenrieder are with the Flexible Electronics Laboratory at the Sensor Technology Research Centre, University of

Sussex Falmer, Brighton, BN19QT, UK (corresponding email: a.pouryazdan@sussex.ac.uk). N. Münzenrieder is also with the Faculty of Science and Technology, Free University of Bozen-Bolzano, 39100 Bozen, Italy.

This work was partially supported by EPSRC, GCRF, and NIHR, under the contact number: EP/R013837/1 (SmartSensOtics)

\begin{abstract}
Capacitive non-contact imaging of electric fields and potentials with micro-metre resolution can provide relevant insights into material characterisation, structural analysis, electrostatic charge imaging and bio-sensing applications. However, scanning electric potential microscopes have been confined to rigid and single-probe devices, making them slow, prone to mechanical damage and complex to fabricate. In this work, we present the design and characterisation of a novel 5-element flexible array of electric potential probes with spatial resolution down to $20 \mu \mathrm{m}$ to speed up the scanning time. This was achieved by combining flexible thin-film probes for active guarding and shielding with state-of-the art discrete conditioning circuits. The potential of this approach is showcased by using the fabricated array to image latent fingerprints deposited on an insulating surface by contact electrification, obtain the surface topography of conductive samples and to visualise local dielectric variations.

Index Terms_Flexible electronics, Capacitive sensors, Electric potential Imaging, Sensor array
\end{abstract}

\section{INTRODUCTION}

$\mathbf{N}$ ON-CONTACT measurements of static and lowfrequency dynamic electric potential and electric fields have many applications in science [2] and engineering, including non-invasive material characterisation [3], electrophysiology [4] and context activity recognition [5], [6]. Measurements of electric potential (voltage) can be divided into two broad categories of contact and non-contact techniques. Contact measurements require resistive connection to the sample under test. Common instruments to measure $\mathrm{AC}$ and DC potential differences are voltmeters, oscilloscopes and amplifiers in general. In contrast, there are various capacitive sensors available to measure electric potentials. A key advantage of capacitive sensing is that measurements can be made without any physical contact. To capacitively measure electric potentials and electric fields voltage amplifiers with virtually infinite input impedance are required. This avoids any disturbance to the field under test and results in a sufficient signal to noise ratio [7].

When measuring electric potential with micro-metre spatial resolution, the required input impedance is beyond the capabilities of conventional measurement systems. Distortion-free measurements require e.g. highly sophisticated MEMS based technologies [8]. Hence, most methods to measure electric potential with micro-scale or better resolution require some sort of physical contact to the sample e.g. contact Atomic Force Microscopy (AFM) [9] or Scanning Capacitance Microscopy (SCM) [10]. However, one approach to overcome this limitation is the use of the capacitive Electric Potential Sensor (EPS) [7]. The ultra-high input resistance of EPS $(\approx 0.1 \mathrm{~T} \Omega)$ along with the ultra-low input capacitance $(\approx 0.3 \mathrm{fF})$ enables the measurements of $\mathrm{AC}$ potentials with frequencies from $50 \mathrm{mHz}$ to $330 \mathrm{MHz}$ and a noise floors as low as $3.5 \mathrm{nV} / \sqrt{\mathrm{Hz}}$ 


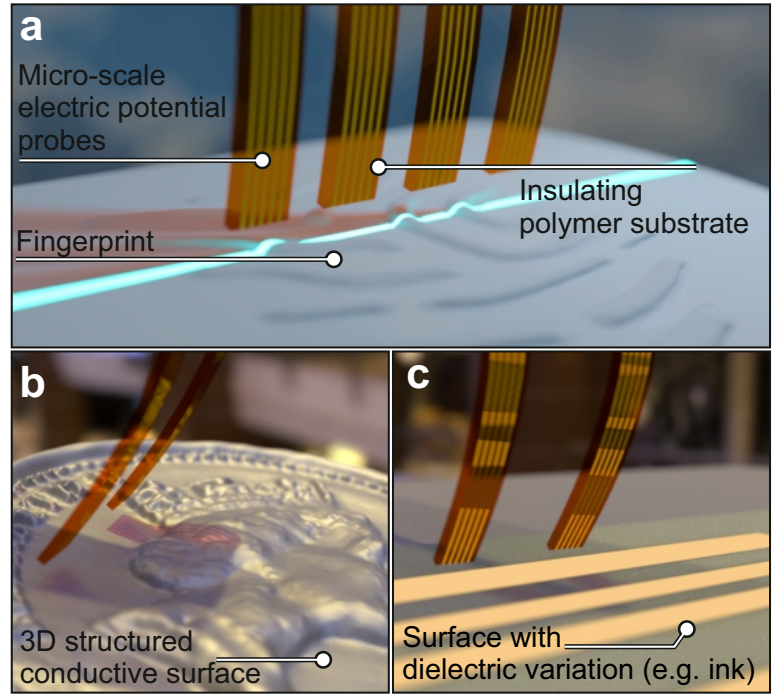

Fig. 1. Concept of electric potential microscopy using a flexible probe array. a) Imaging of electrostatic charge distribution on insulation substrates to reveal latent fingerprints. b) Micro-scale surface topography imaging of conductive samples. c) Spatial imaging of dielectric samples.

[6], [11], [12]. It is also possible to measure DC potentials with microscopic resolution using EPS [13]. The capabilities of EPS technology to image electric fields have been previously demonstrated by integrating such sensors into non-contact scanning electric potential microscopes (SEPM). SEPM can be applied to a plethora of applications in non-destructive material characterisation [3], electrostatic charge imaging [14], measurement of propagation delay on printed circuit boards [15] and localised electro-physiological sensing [16]. The key characteristic of EPS that allows for such measurements is the use positive feedback techniques, active guarding of the sensing probes and special biasing network that makes it DC stable. Thus far, only conventional rigid probes have been used to realize SEPM [17]. Additionally, the scanning process of SEPM can take many hours to image a large area using a single sensing probe. In this context, guarded arrays with micro-scale spatial resolution are highly desirable to reduce the scanning time and cross-talk between the sensing elements. Previously, an eight-element array of EPS was developed using semi-rigid coaxial cables with $2 \mathrm{~mm}$ spatial resolution [15]. BeardsmoreRust et. al. [18] also developed an array of EPS for macroscale electrostatic charge imaging of insulating materials with a spatial resolution of $1 \mathrm{~cm}$. Both of these works lacked the adequate spatial resolution for SEPM applications. However, the advancement of flexible thin-film electronics has opened up the possibility of fabricating active and passive sensor arrays on flexible substrates [1], [19]-[26].

\section{ApproAch}

Here, we present the design, fabrication, characterisation and various operation modes of the flexible 5-element array of EPS sensors for SEPM applications. We provide example data for various operation modes and discuss the advantages and shortfalls of the flexible sensor array in comparison to the traditional single probe SEPM.
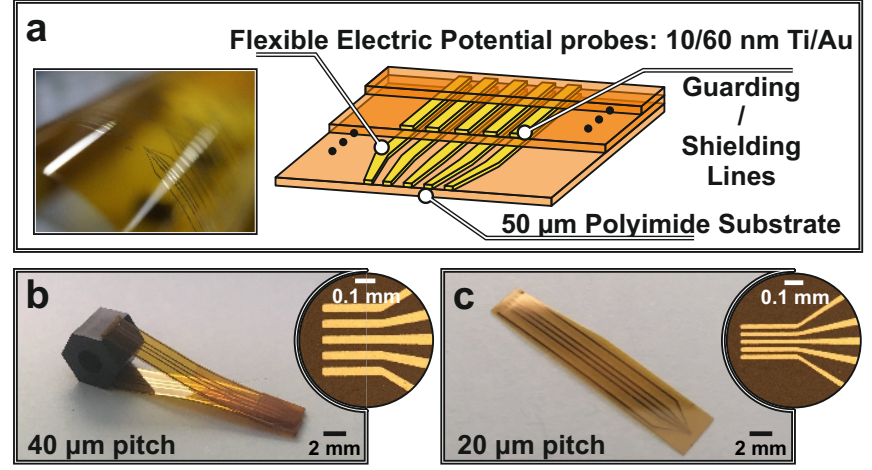

Fig. 2. Arrays of flexible sensing probes fabricated for electric potential microscopy. a) Micro-graph of the fabricated polyimide substrate with 5element SEPM probes and the layer schematic diagram of the probes. $\mathrm{Ti} / \mathrm{Au}$ tracks are deposited on a polyimide substrate and subsequently stacked on top of each other. b) Photograph of the $40 \mu \mathrm{m}$ pitch flexible probes stacked and aligned to provide either active guarding or ground shielding. c) Single layer of 5-element flexible probes with $20 \mu \mathrm{m}$ pitch spacing.

Figure 1 demonstrates the concepts of using the flexible array in three different operation modes. When scanning charged insulating surfaces, the SEPM array is able to image the electrostatic charge distribution of the sample. The EPS array can also be used to obtain the surface topography of conductive samples and to measure the local variation of relative permittivity in dielectric samples as shown in Figure 1b and Figure 1c respectively. To realise this array a hybrid approach is taken where the flexible sensing probes are interfaced to the front-end electronics using conventional off-the-shelf flexible printed circuit connectors (FPC). These connectors are commonly used as a board-to-board connector for thin flexible copper PCBs in space constraint application such as smart rings or virtual reality headsets. The advantages of this approach are manifold: 1) Contrarily to rigid substrates such as glass or silicon, these flexible probes will not damage the substrate in case of contact and are more durable due to their flexibility. 2) The process used to fabricate these probes are large-area compatible and relatively low-cost; 3) The inherent insulating property and low electric permittivity of flexible polymer substrates minimises cross-talk; 4) Finally, we used the low thickness and flexibility of polyimide foil substrates to easily produce overlaying conductive tracks that act as effective shielding or guarding structures, which satisfied the aforementioned requirements of EPS sensing technology. Multiple 5-element sensing arrays with pitch spacing of $40 \mu \mathrm{m}$ and $20 \mu \mathrm{m}$ were fabricated. This work demonstrates that flexible thin-film technology can be readily used to fabricate EPS sensors with micro metre resolution. This is an innovative approach to realise inexpensive, bespoke and large-area capacitively coupled sensor arrays [20]-[26].

\section{Design And Fabrication}

The sensor system was realized using hybrid approach employing flexible thin-film technology and rigid surface mount (SMD) circuitry. 


\section{A. Flexible sensor arrays}

Figure 2a shows an optical micrograph of the presented flexible array. The sensing probe array was fabricated by depositing a $10 \mathrm{~nm}$ Titanium adhesion layer followed by a $60 \mathrm{~nm}$ Gold layer on a $50 \mu \mathrm{m}$ thick polyimide substrate through e-beam evaporation. Gold was chosen since it does not easily oxidise and provides both high conductivity and ductility. In addition, $60 \mathrm{~nm}$ Gold thin-films were used since this thickness provides a good trade-off between cost, conductivity and connector stability. These metal layers were then patterned using standard photolithography and lift-off. A schematic of the layers structure is illustrated in Figure 2a. The sensing probe array is then shielded or guarded by stacking and aligning another identical thin-film structure on top of the sensing probe array. The two thin-film arrays are then bonded together by wrapping a Kapton tape around them. Using these methods, arrays of 5 independent sensing electrodes with pitch sizes of $40 \mu \mathrm{m}$ and $20 \mu \mathrm{m}$ were fabricated on one substrate. These reduced pitch sizes were possible given the polyimide's low surface roughness $(<4 \mathrm{~nm})$, good electric insulating properties and compatibility with photolithography techniques. The arrays can be guarded/shielded or un-shielded as shown in Figure 2c and Figure 2d respectively. The spatial resolution is defined by the width of each conductive sensing element on the flexible probe array and the pitch spacing between them.

\section{B. Conditioning circuit}

The flexible sensing probes are conditioned using a high impedance circuit made from discreet components on a $1 \mathrm{~mm}$ thick FR4 printed circuit board. Figure 3a shows an image of the conditioning circuit board for all five sensing elements. Figure $3 \mathrm{~b}$ shows a close-up of a commercially available FPC connector with $600 \mu \mathrm{m}$ pitch spacing used to interface the flexible sensing probes and their corresponding flexible guarding or shielding structure to the conditioning circuits. The complete assembly results in a 5-element SEPM sensor array enclosed in a grounded metal box that acts like a Faraday cage. Figure 4a shows an equivalent circuit schematic diagram of the EPS sensor technology. $C_{\text {coupling }}$ represents the capacitive coupling between each sensing probe and the sample under test and is defined by the sensing probe area and its separation to the sample. $C_{i n}$ and $R_{i n}$ represent the internal input capacitance and the input resistance of the EPS conditioning circuit. $C_{i n}$ forms a capacitive voltage divider network with $C_{\text {coupling }}$ and $R_{\text {in }}$ forms a first order high-pass filter with the coupling capacitance, both of which can decreases sensitivity. By use of positive feedback the input impedance $\left(Z_{i n}\right)$ is enhanced which in turn increases the sensitivity. High sensitivity is essential to achieve high spatial resolution, since the sensed signal strength is inversely proportional to the size of the probes and sample to sensor separation. Here the higher sensitivity is attained by neutralising any parasitic capacitance between the high impedance sensing probes to ground. $R_{i n}$ can also be artificially increased to improve the lower cut-off point of the frequency response. Depending on the sample under test, either active guarding or passive shielding is implemented. Active guarding is a form of positive feedback. The active guarding topology is shown in Figure 4b, whereas the shielding topology is shown in Figure 4c. While guarding increases the sensitivity, it can also increase crosstalk between each element in an array of sensors as discussed in the next section. When the electric potential to be measured is large, active guarding is replaced in favor of shielding at the cost of lower sensitivity.

\section{Positioning system}

The complete sensor assembly is mounted on a computer controlled XYZ positioning system for characterisation and scanning electric potential measurements. The three-axis positioning system used here is a re-purposed PCB milling machine which has a resolution of $6.2 \mu \mathrm{m}$ in $\mathrm{X}$ and $\mathrm{Y}$ axes. It has an increment of $625 \mathrm{~nm}$ in Z-axis for precise adjustment of the sensing probe to sample separation. All three axes are driven by open-loop stepper motors with $1.8^{\circ}$ rotational angle configured to rotate at quarter-steps using Toshiba TB67S109 chopper-type ICs. The motor drivers are connected to a micro controller unit (MCU) which is connected to a PC. The MCU handles positioning commands received from a bespoke LabView virtual instrument interface on the PC. The LabView VI is also responsible for acquiring, visualising and storing the digitised sensor outputs on the PC. Before the sensor outputs are digitised using a National Instrument NI-6210 USB acquisition device the signals are filtered using 4th order Butter-worth high-pass and low-pass filters to reduce scanning artefacts and any other extrinsic noise pick-up.

\section{Performance and characterisation}

The fully assembled sensor system was characterised under ambient conditions in an un-shielded environment using a HP 3562A dynamic signal analyser. The frequency response, spatial resolution and cross-talk between each sensing element within the array were evaluated. The noise floor of the frontend conditioning circuit is the most important parameter in non-contact SEPM as it defines the minimum detectable signal. Figure 5 shows the voltage noise spectral density of one of the conditioning circuits when the input was shorted to ground through a $1 \mu \mathrm{F}$ capacitor. The $1 / \mathrm{f}$ corner is at $40 \mathrm{~Hz}$. Above this frequency the noise level is $4.8 \mathrm{nV} / \sqrt{\mathrm{Hz}}$ refereed to input.

\section{A. Frequency response}

The frequency response for all five sensors was measured one at a time as shown in Figure 6. The measurement was carried out with each sensor being once resistively (DC) coupled and another time capacitively (AC) coupled (through a calibrated $1 \mathrm{pF}$ capacitor) to the source generator of the dynamic signal analyser. As shown in Figure 6a all five sensors have similar responses when DC coupled. The sensors are designed to have a DC voltage gain of $23 \mathrm{~V} / \mathrm{V}(27.3 \mathrm{~dB})$. The higher noise levels at lower frequencies can be attributed to the vibration in the sensing probes and cabling connecting the sensor array to the signal analyser.

However, the sensors have different frequency response when AC coupled (Figure $6 \mathrm{~b}$ ). This is due to the change in 

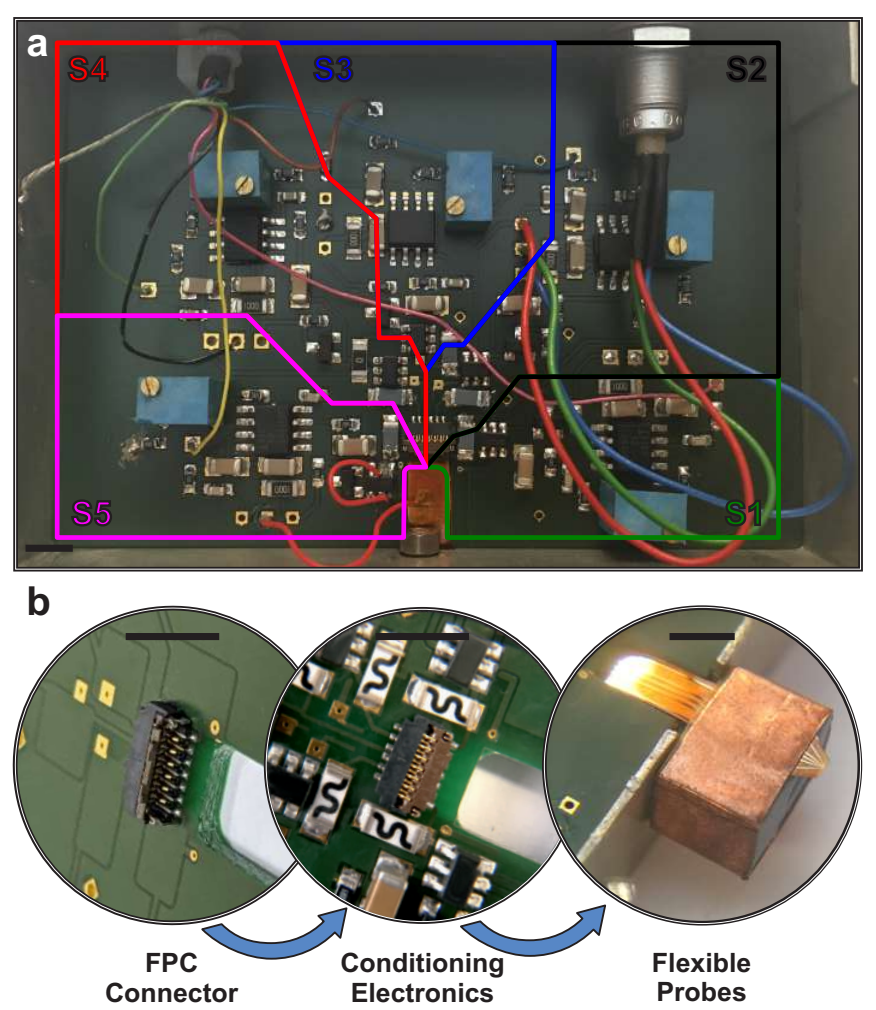

Fig. 3. Assembly of 5-element SEPM array. a) Photograph of the conditioning circuit used to read out the signals form the flexible probes. b Assembly of the flexible sensing array interfaced to the front-end conditioning circuits using FPC connectors. All scale bars are $5 \mathrm{~mm}$.

layout on both the flexible sensor array and on the sensor conditioning PCB. Specifically, sensor 1 and 5 differ to the rest of the sensing elements. Sensors 1 and 5 are both routed on the edge of the flexible probe structure and have twice the pitch spacing $(1.2 \mathrm{~mm})$ to their adjacent sensing elements at the FPC connector end. The variation in spacing and layout changes the capacitive coupling of the high impedance sensing traces to their surroundings and resulted in different sensitivity levels. Furthermore, the sensors have lower voltage gains or sensitivity when AC coupled. This is due to the capacitive voltage divider network formed between $C_{\text {Coupling }}$ and $C_{i n}$. The noise spikes around $50 \mathrm{~Hz}$ and its harmonics are unwanted interference pick-up from nearby 240 mains power. $6 \mathrm{~b}$ also shows that the sensors have a lower cut-off point of $\approx 2 \mathrm{~Hz}$ and an upper bandwidth limit of $\approx 100 \mathrm{kHz}$.

\section{B. Cross-talk}

Two methods were used to characterise the cross-talk between each element of the sensor array, namely, spatial crosstalk and spectral cross-talk as described below.

1) Spatial cross-talk: Figure 7 shows an schematic of the measurement setup and the results used to quantify the spatial cross-talk for various shielding and guarding configurations. A $15 \mu \mathrm{m}$ insulated wire was placed on a grounded copper plane. A $10 \mathrm{kHz}$ AC voltage signal with an amplitude of $1 \mathrm{~V}$ was applied between the wire and the copper plane. The sensor array was then moved over this wire horizontally at a constant height of $100 \mu \mathrm{m}$ above the wire while the outputs of all
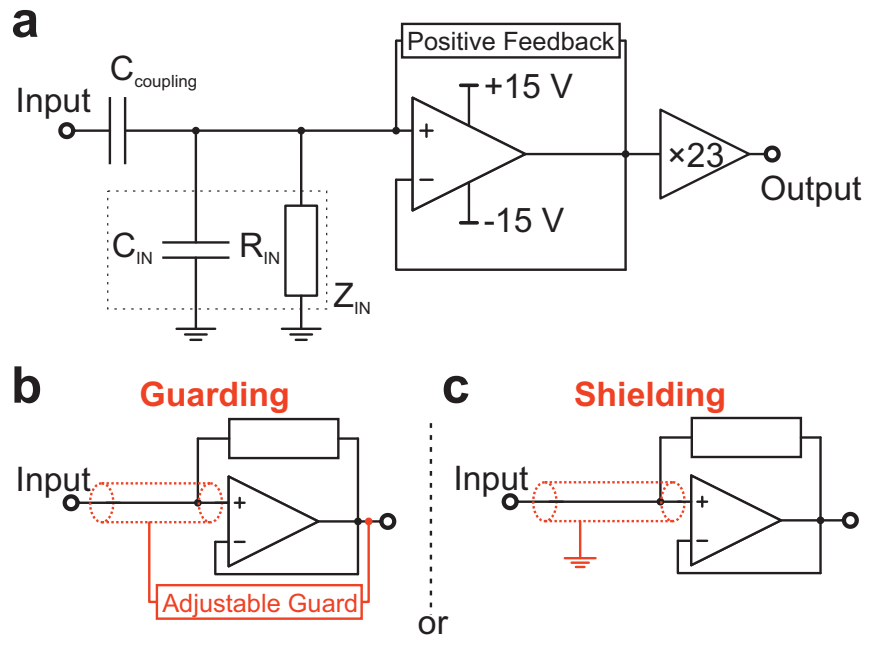

Fig. 4. Equivalent circuit representation of a single conditioned sensor. a) Schematic diagram of the EPS sensor technology. $\boldsymbol{C}_{\text {coupling }}$ is the capacitive coupling between the sensing probe and a sample under test. $\boldsymbol{C}_{\boldsymbol{i n}}$ and $\boldsymbol{R}_{\boldsymbol{i n}}$ represent the input capacitance and the input resistance of the EPS conditioning circuit. b) Active guarding and c) Ground shielding of the sensing probes.

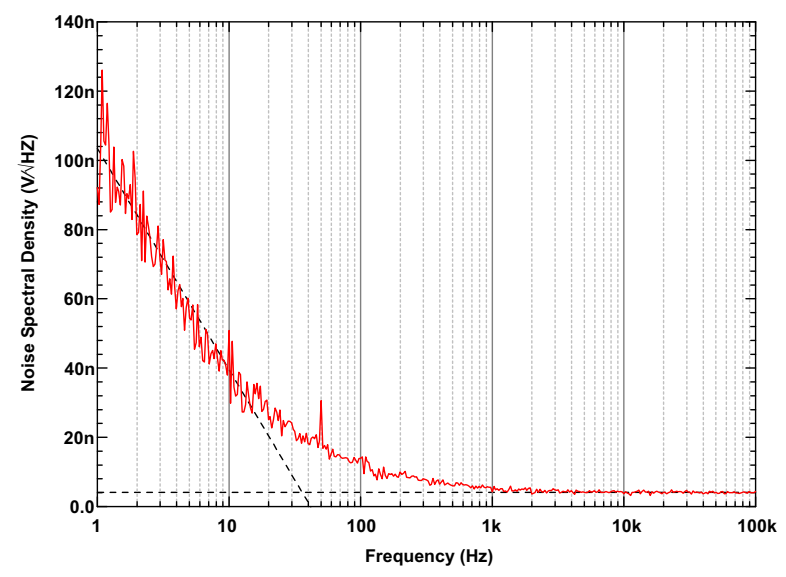

Fig. 5. Voltage noise spectral density of one of five input condition circuits. obtained using the HP $3562 \mathrm{~A}$. Above the $1 / \mathrm{f}$ corner frequency of $40 \mathrm{~Hz}$, the noise level is flat at $4.8 \mathrm{nV} / \sqrt{\mathrm{Hz}}$ refereed to input.

five sensors were recorded. This is illustrated in Figure 7a. Multiple guarding and shielding configurations were tested and the results from each is shown in Figures $7 \mathrm{~b}$ to $7 \mathrm{e}$. As shown, ground shielding or no shielding at all were the most effective configurations at minimising cross-talk (Figures $7 \mathrm{~b}$ and $7 \mathrm{c}$ ). However, ground shielding reduces sensitivity, while lack of shielding introduces interference from unwanted electric field sources such as the mains $50 \mathrm{~Hz}$ signal. For the guarded double layer structure shown in Figure $7 \mathrm{~d}$, the cross-talk increases with the amplitude of the guard signal. While active guarding can increase sensitivity, above a certain level the guard signal also increases the cross-talk beyond a usable condition. This can be attributed to capacitive coupling of the guard signals from the adjacent sensors through the PCB and the Polyimide flexible probe. To maintain both a high sensitivity and guarding against unwanted noise sources, the active guard amplitude was reduced to its minimum (Figure 7e). 

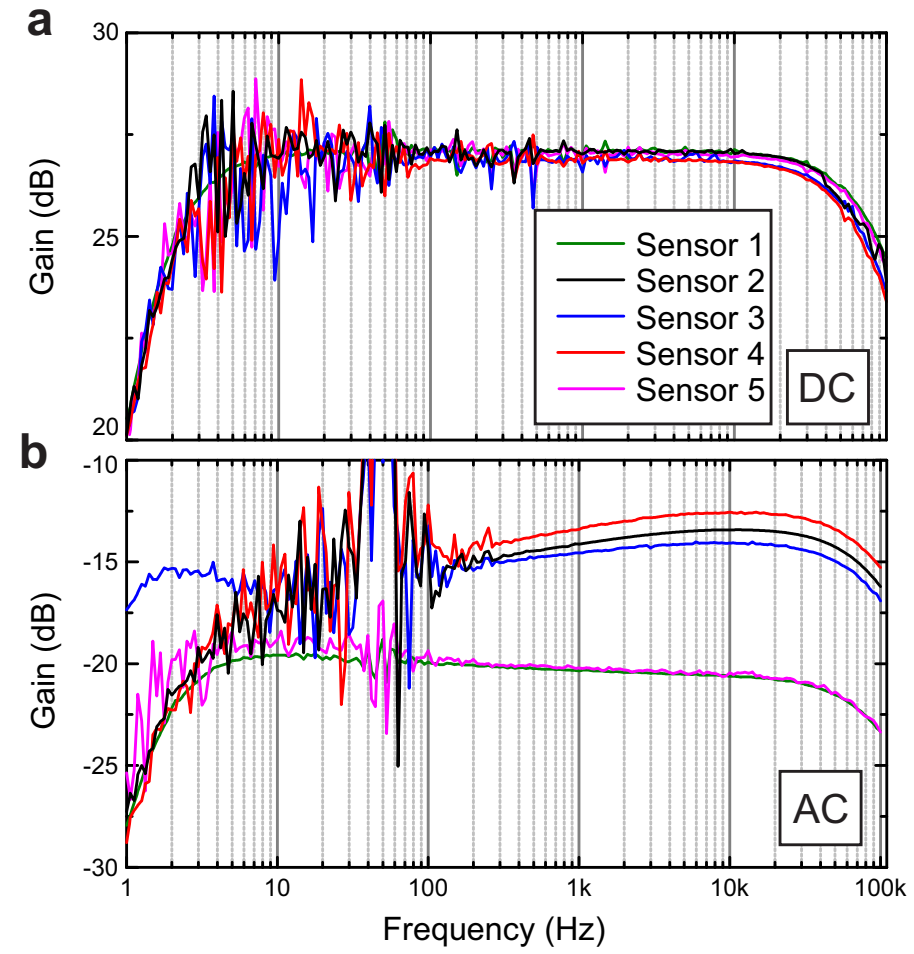

Fig. 6. Frequency response of the sensor array. a) Each element in the sensor array was DC coupled to the spectrum analyser. b) Spectrum with the array $\mathrm{AC}$ coupled to the spectrum analyser through a $1 \mathrm{pF}$ capacitor.

2) Spectral cross-talk: Another approach to characterise the cross-talk is to apply different frequencies to each sensing element and compare the amount of spectral leakage in other sensors. This is also illustrated in Figure 8a. The sources were fed to the the sensor using the same Polyimide structure as the sensing probe. Figure $8 \mathrm{c}$ shows the measurements for the sensing probes resistively coupled to the AC sources. As expected, the spectral leakage to the adjacent sensors is almost negligible in this case as the sensors are driven by a lowimpedance source. However, when the sensing elements were separated by a distance of $100 \mu \mathrm{m}$ from the source (i.e. AC or capacitively coupled) the spectral leakage to the adjacent sensors increases (Figure 8d). This is due to the unwanted capacitive coupling of the high impedance sensing probes to the adjacent sources. Here, the active guarding configuration shown in Figure 7e was chosen to carry out this measurement. Figure 8d shows that the three sensing probes in the middle with $0.6 \mathrm{~mm}$ pitch spacing have more cross-talk in comparison to the two outer probes which have double the pitch spacing at the FPC end. However, the isolation between the channels is adequate as to distinguish the designated signal emitting from the sources under each sensing element. The isolation between channels is visualised with the aid of a confusion matrix shown in Figure 8b. The orthogonal of the matrix shows the isolation between each sensor to the rest of the array. The highest isolation is between the two furthest sensing probes as expected.

\section{A. Electrostatic charge distribution}

Spatial imaging of electrostatic charge distribution is classified as a passive operation mode. Figure 9a shows the principles of this operation mode. The sensing probes are scanned over a charged surface at a constant sample to probe separation of $\approx 150 \mu \mathrm{m}$. The presence of electrostatic charges induces a voltage on the conductive sensing probe of the array. The voltage measured is proportional to the density of these electro-static charges. These voltage are read by the high impedance signal conditioning front-end circuitry. The amplitude of the signals are proportional to the electric charge and the frequency of this time varying signal is a function of the scanning speed. A detailed description of this measurement mode is described elsewhere [1], [14]. Even though the Polyimide substrate of the sensor array can collect electrostatic surface charges, these charges have no influence on the measurement since they move with the array itself. Furthermore, before the measurement is performed, the probes are deionised using a bench-top zero Volt $5 \mathrm{kV}$ air de-ioniser device. An immediate application for measuring spatial electrostatic charge distribution is the the imaging of latent fingerprint deposited on insulating materials due to contact electrification of two dissimilar materials. This was previously shown using a single ridge sensing probe [27]-[29]. Figure 10a shows an optical image of a fingerprint. The very same fingerprint was also deposited on a $75 \mu \mathrm{m}$ PTFE (Polytetrafluoroethylene) film by contact electrification. Figure 10b shows the electrostatic image of the fingerprint obtained using a single sensor while Figure 10c shows the image measured using the presented flexible 5-element sensor array. The two micrographs are comparable, except for the vertical scanning scar lines visible in Figure 10c. The lines appear since the sensors do not have the exact same performance. To compensate for the difference in $\mathrm{AC}$ sensitivity of the sensors, the outputs of the sensors were normalised. The full scan of the fingerprint using the five-element sensor array reduced the measurement time by a factor of five from $25 \mathrm{~min}$ to $5 \mathrm{~min}$. The reduced scanning 

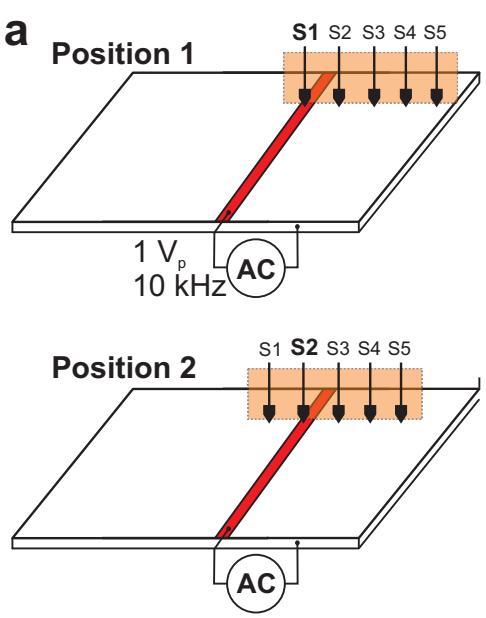

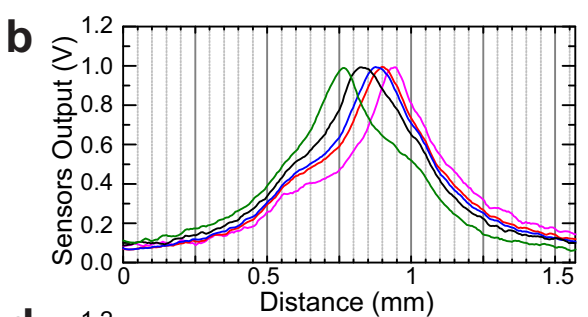

d

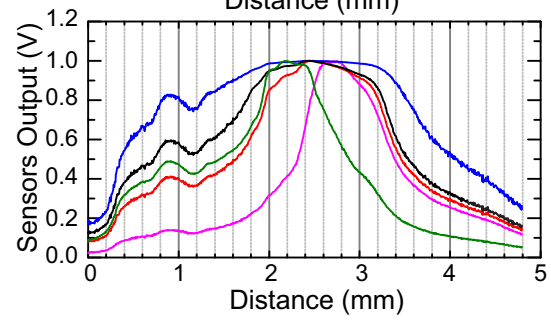

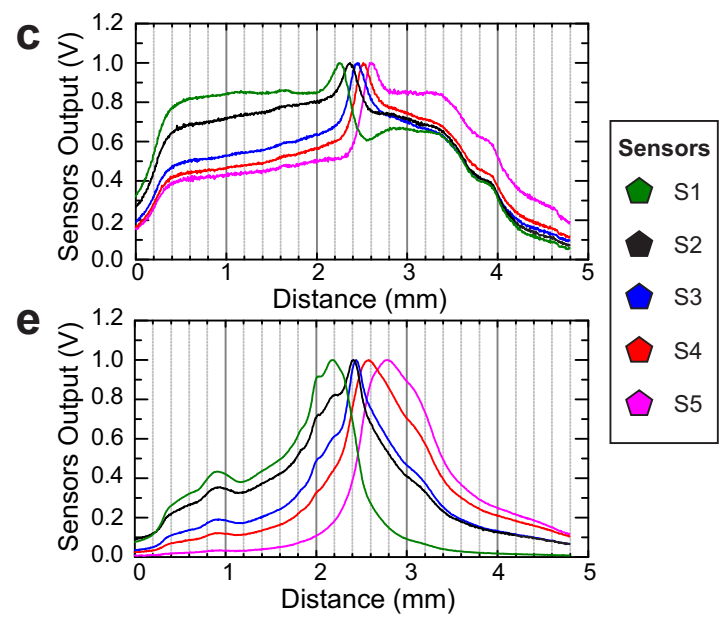

Fig. 7. Spatial cross-talk characterisation of the five-element array. a) Schematic of the setup utilised to study the cross-talk between the micro-scale probes. b) $20 \mu \mathrm{m}$ pitch ground shielded probe. c) $40 \mu \mathrm{m}$ pitch single layer un-shielded probe. d) $40 \mu \mathrm{m}$ stacked guarded electrode with maximum active guarding and e) $40 \mu \mathrm{m}$ stacked guarded electrode with minimum active guarding.

a
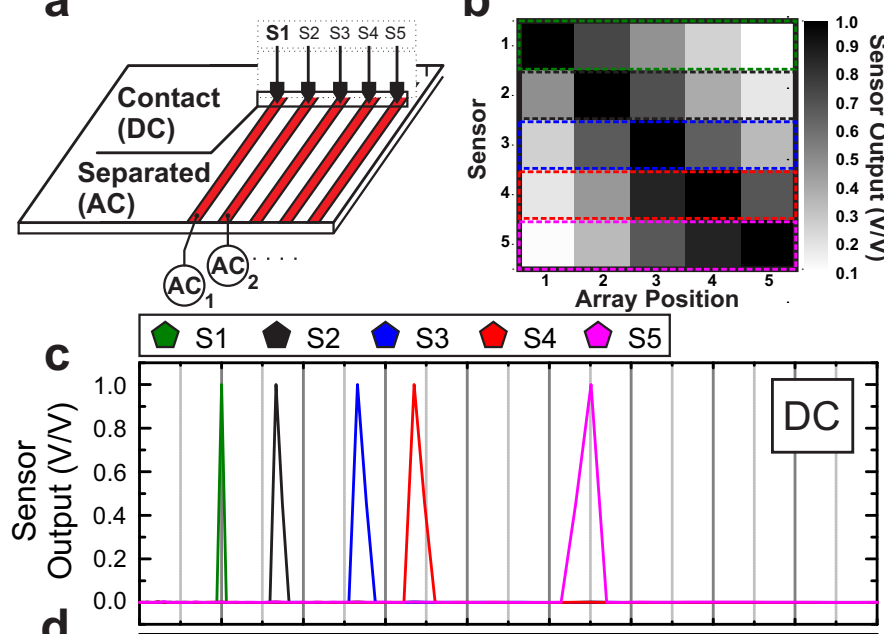

d

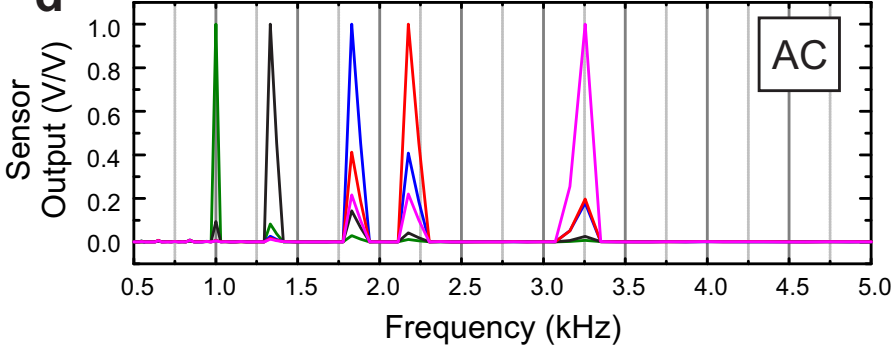

Fig. 8. Spectral cross-talk characterisation. a) Schematic diagram of the measurement setup. b) Sensor array resistively coupled to the sources and c) sensor array capacitively coupled to the sources.

time together with the close match between optical and SEPM imaging have significant benefits for forensic applications.

\section{B. Surface topography}

Another operation mode of SEPM is imaging of the surface topography of conductive samples. This is an active measurement as an AC signal is applied to the sample under test. Figure $9 \mathrm{~b}$ shows the schematic diagram for this measurement mode. The amplitude of the excitation signal measured by

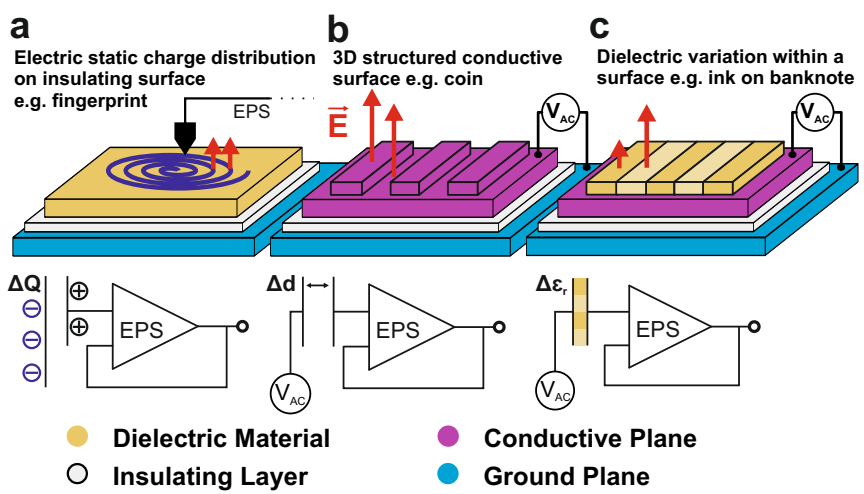

Fig. 9. Various possible operation modes for SEPM. a) Imaging of spatial electrostatic charge distribution on dielectric materials. b) Surface topography measurement of conductive samples. c) Spatial imaging of dielectric variation within a surface.

the sensing probes varies with the change in distance to the sample due to the samples feature. This is noted as $\Delta \mathrm{d}$ in the schematic. To confirm the functionality of the presented array for SEPM based surface topography, the surface of a 20 pence coin (Figure 10d) was mapped. A $1 \mathrm{~V} \mathrm{AC}$ voltage signal of $10 \mathrm{kHz}$ was applied to the coin and a grounded copper plane below it separated by an insulator (paper). The ground surface below the paper ensures that the coin is not at an arbitrary potential. Hence the sensed amplitude of the excitation signal will vary with the features of the sample under test. In other words, the variation in the measured signal is due to the separation between the sample features and the sensor tip or the capacitive coupling between the two. Figure 10e shows the results obtained using the presented flexible sensor array while Figure 10f shows the single ridged sensing probe counterpart. Similar to the electrostatic charge mapping, the measurement time was reduced by a factor of five by use of the presented 5-element sensor array for the surface topography, without significant impact on the image quality. 


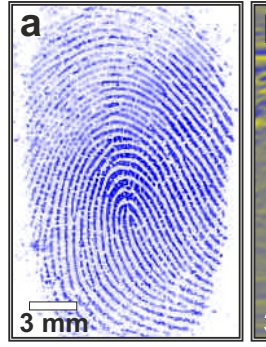

Sensors Output (V)

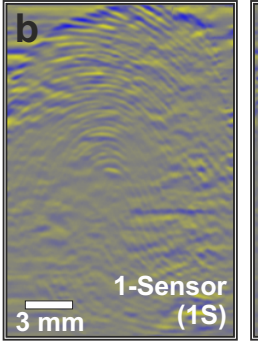

(1S)

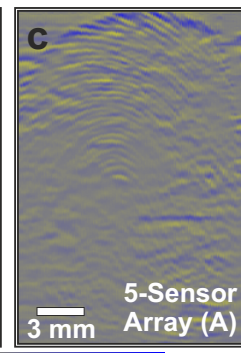

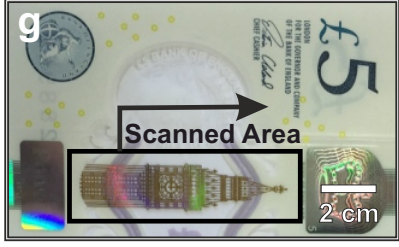

Sensors Output (V)

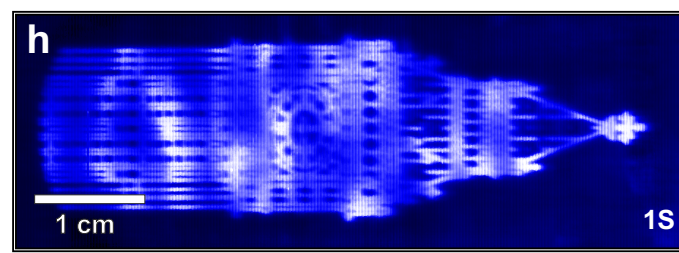

$1 \mathrm{~cm}$

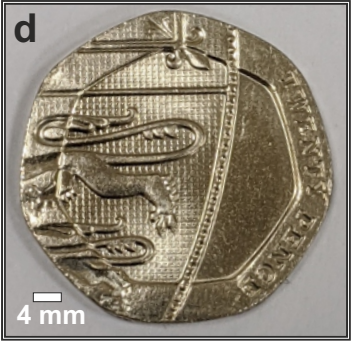

Sensors Output (V)

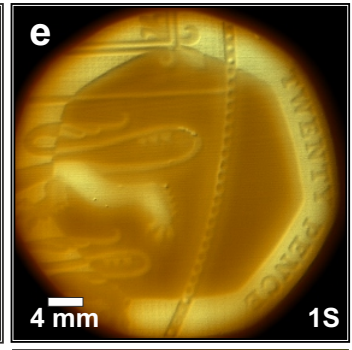

15

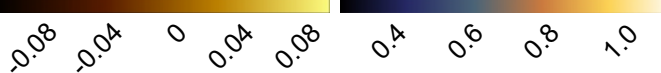

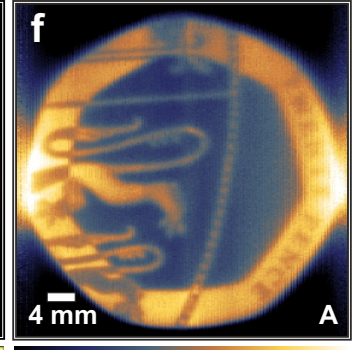

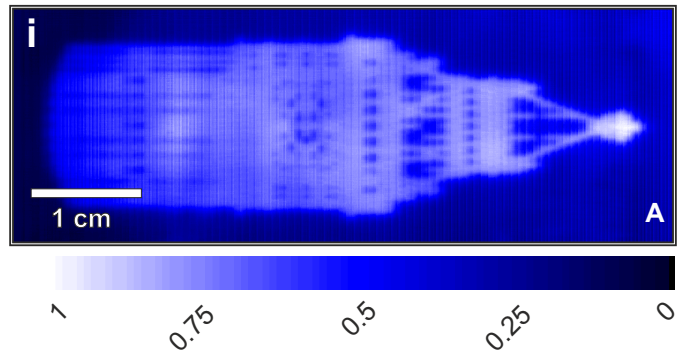

Fig. 10. Electric potential imaging using the flexible sensor array. a) Optical image of a fingerprint. Electrostatic imaging of the fingerprint using b) A single probe and c) the presented 5-element flexible array. d) Optical image of a 20 pence coin used as an example of a conductive sample and e) the corresponding surface topography of the coin acquired using the 5-element flexible array. f) single probe measurement of the $20 p$ coin. g) Optical photo a 5 Pounds plastic bank note used as a dielectric sample. h) The corresponding SEPM imaging of the bank note using a single probe and i) using the 5-element sensor array.

\section{Dielectric variation}

Scanning electric potential microscopy can also be used to image the variation in relative permittivity $\left(\Delta \epsilon_{r}\right)$ of dielectric surfaces. The schematic diagram for this mode is shown in Figure 9c. This is another active mode of operation where an AC excitation voltage is applied to a plane under the sample. Here, a genuine plastic five pounds banknote was used as an example of a composite dielectric surface (Figure 10g). Various inks and materials utilised to produce this banknote have different dielectric properties. By measuring the local variation in the amplitude of the $1 \mathrm{~V} 10 \mathrm{kHz}$ AC excitation voltage traveling through the bank note, an electrical image of the note was produced based on its varied local dielectric properties. The permittivity of each material in the bank note alters the electric potential travelling though it and hence the local amplitude of the sensed excitation signal will vary at each measured point. Increasing the amplitude of the excitation signal can compensate for thicker samples. The presented flexible sensing probe array was used to image the marked area of the banknote (Figure 10i). The same area was also measured using a single ridged probe image for comparison purposes. This is show in Figure $10 \mathrm{~g}$. Compared to the single rigid sensing probe, the image produced by the array has lower spatial resolution. This is mainly due to the fact that the Polyimide based sensor array has to be separated from the surface of the dielectric sample further to avoid physical contact; as any contact between the two dielectric samples can cause electrostatic charging of the sample due to contact electrification. Electrostatic charges can induce large voltage on the sensing probes an interfere with the amplitude of the excitation signal being measured. The increased in separation between the sample and the sensor array also reduces the capacitive coupling between the two which in turn degrades the signal to noise ratio. Similar to the other measurement modes, the use of the 5-element array has reduced the measurement time by a factor of five but this time at the cost of reduced sensitivity and spatial resolution.

\section{CONCLUSION}

We presented a proof of concept and confirmed the feasibility of using thin-film technology and conventional silicon based active components to realize a microscopic array of electric potential sensors for SEPM application. The use of the 5-element sensor array reduced the measurement time by a factor of five. This technique can be scaled up to have a higher number of sensing elements in an array. Here, we fabricated a five-element array merely as a proof of concept. We found that by stacking thin-film structures it is possible to achieve active guarding of the sensing probes. For applications such as imaging of electrostatic charge distribution, where large voltages $(\mathrm{kV})$ are measured, a simple ground shield provides adequate cross-talk protection at the cost of reduced sensitivity. Guarding should only be used when a high sensitivity is required as this method of guarding increases cross-talk interference. Due to the large-area compatible fabrication process of the presented flexible and mechanically durable sensor array, these can be fabricated inexpensively on bio-compatible polymeric substrates. The use of FPC connectors enables swift reconfiguration of the guarding/shielding as well as replacing the probes to achieve various spatial resolutions. In the future, we envision stacking multiple 1D arrays of these sensors to achieve a 2D array. Additionally the size and pitch spacing of the sensing probes can be reduced to achieve a higher spatial resolution. The presented technique can be extended to take advantage of the bendable and stackable nature of 
the thin-film probes to implement a two dimensional array suitable for SEPM applications. With further advancement in thin-film transistor technology, and the use of innovative and efficient readout typologies [30] it will be possible to integrate a complete SEPM sensing system including the frontend conditioning circuitry on a single flexible substrate.

\section{REFERENCES}

[1] A. Pouryazdan, J. C. Costa, P. Lugoda, R. J. Prance, H. Prance, and N. Münzenrieder, "Flexible micro-scale sensor array for non-contact electric potential imaging," in 2020 IEEE International Conference on Flexible and Printable Sensors and Systems (FLEPS). IEEE, 2020, pp. $1-4$.

[2] K. Kamiya, K. Ida, M. Yoshinuma, C. Suzuki, Y. Suzuki, M. Yokoyama, L. E. Group et al., "Characterization of edge radial electric field structures in the large helical device and their viability for determining the location of the plasma boundary," Nuclear Fusion, vol. 53, no. 1, p. 013003, 2012

[3] S. Mukherjee, P. Watson, and R. Prance, "Non-contact measurement of local conductivity variations in carbon fibre based composite materials," in Journal of Physics: Conference Series, vol. 307, no. 1. IOP Publishing, 2011, p. 012034.

[4] N. Steinhausen, H. Prance, and R. Prance, "Human computer interface based on eye movement (eog) signals," in Proceedings of AAATE, 2011.

[5] T. Grosse-Puppendahl, X. Dellangnol, C. Hatzfeld, B. Fu, M. Kupnik, A. Kuijper, M. R. Hastall, J. Scott, and M. Gruteser, "Platypus: Indoor localization and identification through sensing of electric potential changes in human bodies," in Proceedings of the 14th Annual International Conference on Mobile Systems, Applications, and Services. ACM, 2016, pp. 17-30.

[6] H. Prance, P. Watson, R. Prance, and S. Beardsmore-Rust, "Position and movement sensing at metre standoff distances using ambient electric field," Measurement Science and Technology, vol. 23, no. 11, p. 115101, 2012.

[7] R. Prance and C. Harland, "Electric potential sensor," Nov. 8 2011, uS Patent 8,054,061.

[8] A. Kainz, H. Steiner, J. Schalko, A. Jachimowicz, F. Kohl, M. Stifter, R. Beigelbeck, F. Keplinger, and W. Hortschitz, "Distortion-free measurement of electric field strength with a mems sensor," Nature electronics, vol. 1, no. 1, p. 68, 2018.

[9] S. Decossas, J. Marchand, and G. Bremond, "Electrical characterisation of local electronic properties of self-assembled semiconductor nanostructures using afm," Physica E: Low-dimensional Systems and Nanostructures, vol. 23, no. 3, pp. 396-400, 2004.

[10] C. Nakakura, D. Hetherington, M. Shaneyfelt, P. Shea, and A. Erickson, "Observation of metal-oxide-semiconductor transistor operation using scanning capacitance microscopy," Applied physics letters, vol. 75, no. 15 , pp. 2319-2321, 1999.

[11] A. Pouryazdan, "Non-contact single-ended electric potential measurements; techniques and applications," PhD Thesis, University of Sussex, 2019.

[12] A. Pouryazdan, J. C. Costa, R. J. Prance, H. Prance, and N. Munzenrieder, "Non-contact long range AC voltage measurement," in 2019 IEEE SENSORS. IEEE, Oct. 2019, pp. 16-4.

[13] A. Pouryazdan, J. C. Costa, F. Spina, R. J. Prance, H. Prance, and N. Münzenrieder, "Non-contact measurement of dc potentials with applications in static charge imaging," in 2020 IEEE SENSORS. IEEE, 2020, pp. 1-4.

[14] P. Watson, R. J. Prance, S. T. Beardsmore-Rust, and H. Prance, "Imaging electrostatic fingerprints with implications for a forensic timeline," Forensic science international, vol. 209, no. 1, pp. e41-e45, 2011.

[15] W. Gebrial, R. Prance, T. Clark, C. Harland, H. Prance, and M. Everitt, "Noninvasive imaging of signals in digital circuits," Review of Scientific Instruments, vol. 73, no. 3, pp. 1293-1298, 2002.

[16] C. Harland, T. Clark, N. Peters, M. J. Everitt, and P. Stiffell, "A compact electric potential sensor array for the acquisition and reconstruction of the 7-lead electrocardiogram without electrical charge contact with the skin," Physiological measurement, vol. 26, no. 6, p. 939, 2005.

[17] A. Clippingdale, R. Prance, T. Clark, and F. Brouers, "Non-invasive dielectric measurements with the scanning potential microscope," Journal of Physics D: Applied Physics, vol. 27, no. 11, p. 2426, 1994.
[18] S. Beardsmore-Rust, P. Watson, R. Prance, C. Harland, and H. Prance, "Imaging of charge spatial density on insulating materials," Measurement Science and Technology, vol. 20, no. 9, p. 095711, 2009.

[19] J. C. Costa, F. Spina, P. Lugoda, L. Garcia-Garcia, D. Roggen, and N. Münzenrieder, "Flexible sensors-from materials to applications," Technologies, vol. 7, no. 2, p. 35, 2019.

[20] E. Pritchard, M. Mahfouz, B. Evans, S. Eliza, and M. Haider, "Flexible capacitive sensors for high resolution pressure measurement," in Sensors, 2008 IEEE, 2008, pp. 1484-1487.

[21] C. Metzger, E. Fleisch, J. Meyer, M. Dansachmüller, I. Graz, M. Kaltenbrunner, C. Keplinger, R. Schwödiauer, and S. Bauer, "Flexible-foam-based capacitive sensor arrays for object detection at low cost," Applied Physics Letters, vol. 92, no. 1, p. 013506, 2008

[22] Y.-J. Yang, M.-Y. Cheng, W.-Y. Chang, L.-C. Tsao, S.-A. Yang, W.-P. Shih, F.-Y. Chang, S.-H. Chang, and K.-C. Fan, "An integrated flexible temperature and tactile sensing array using pi-copper films," Sensors and Actuators A: Physical, vol. 143, no. 1, pp. 143-153, 2008.

[23] M. Cheng, X. Huang, C. Ma, and Y. Yang, "A flexible capacitive tactile sensing array with floating electrodes," Journal of Micromechanics and Microengineering, vol. 19, no. 11, p. 115001, 2009.

[24] T. Someya, T. Sekitani, S. Iba, Y. Kato, H. Kawaguchi, and T. Sakurai, "A large-area, flexible pressure sensor matrix with organic field-effect transistors for artificial skin applications," Proceedings of the National Academy of Sciences of the United States of America, vol. 101, no. 27, pp. 9966-9970, 2004

[25] F. Jiang, G.-B. Lee, Y.-C. Tai, and C.-M. Ho, "A flexible micromachinebased shear-stress sensor array and its application to separation-point detection," Sensors and Actuators A: Physical, vol. 79, no. 3, pp. 194 203, 2000.

[26] H. Fang, K. J. Yu, C. Gloschat, Z. Yang, E. Song, C.-H. Chiang, J. Zhao, S. M. Won, S. Xu, M. Trumpis et al., "Capacitively coupled arrays of multiplexed flexible silicon transistors for long-term cardiac electrophysiology," Nature Biomedical Engineering, vol. 1, p. 0038, 2017.

[27] P. Watson, R. Prance, H. Prance, and S. Beardsmore-Rust, "Imaging the time sequence of latent electrostatic fingerprints," SPIE-Optics and Photonics for Counterterrorism and Crime Fighting VI and Optical Materials in Defence Systems Technology VII, pp. 783 803-1, 2010.

[28] J. Lowell and A. Rose-Innes, "Contact electrification," Advances in Physics, vol. 29, no. 6, pp. 947-1023, Dec. 1980.

[29] D. J. Lacks and R. M. Sankaran, "Contact electrification of insulating materials," Journal of Physics D: Applied Physics, vol. 44, no. 45, p. 453001 , Oct. 2011

[30] J. C. Costa, A. Pouryazdan, R. J. Prance, H. Prance, and N. Münzenrieder, "Flexible bootstrapped cascode system with feedback for capacitive through-substrate electric potential measurements with a 55 db relative gain," in 2020 International Electron Devices Meeting. IEEE, 2020, pp. 1-4. 